\title{
ON THE INDIVIDUAL ERGODIC THEOREM FOR POSITIVE OPERATORS
}

\author{
RYOTARO SATO
}

\begin{abstract}
A theorem which gives a condition on a positive linear contraction on an $L^{1}$-space in order that the individual ergodic theorem hold is proved. The theorem contains a result obtained by $\mathrm{Y}$. Ito as a special case.
\end{abstract}

Let $(X, \mathscr{M}, m)$ be a $\sigma$-finite measure space and let $T$ be a positive linear contraction on $L^{1}(m)$. Let $a_{n, j}$ be a matrix of numbers such that

$$
\begin{gathered}
\sum_{j=0}^{\infty}\left|a_{n, j}\right|<\infty \text { for } n=0,1, \cdots, \\
\lim _{n^{\prime}} \sum_{j=0}^{\infty} a_{n^{\prime}, j}=1, \\
\lim _{n^{\prime}} \sum_{j=0}^{\infty} a_{n^{\prime}, j} b_{j+1}=b
\end{gathered}
$$

whenever $b_{0}, b_{1}, \cdots$ is a bounded sequence of numbers for which $\lim _{n^{\prime}} \sum_{j=0}^{\infty} a_{n^{\prime}, j} b_{j}=b$ exists and is finite, where $\left\{n^{\prime}\right\}$ is a subsequence of $\{n\}$.

Under these conditions we shall prove the following

THEOREM. If there exists a strictly positive function $h$ in $L^{1}(m)$ such that the set $\left\{\sum_{j=0}^{\infty} a_{n, j} T^{j} h ; n \geqq 0\right\}$ is weakly sequentially compact in $L^{1}(m)$, then for any $f \in L^{1}(m)$ the limit

$$
\lim _{n} \frac{1}{n} \sum_{j=0}^{n-1} T^{j} f(x)
$$

exists and is finite almost everywhere.

The following proof is a generalization of that given by $Y$. Ito in [6]. Proof. Let $g \in L^{1}(m)$ and $\left\{n^{\prime}\right\}$ a subsequence of $\{n\}$ such that

Received by the editors March 23, 1972.

AMS 1970 subject classifications. Primary 47A35.

Key words and phrases. Positive linear contraction, individual ergodic theorem, weak sequential compactness.

(C) American Mathematical Society 1973 
$\sum_{j=0}^{\infty} a_{n^{\prime}, j} T^{j} h$ converges weakly to $g$. Then for any $u \in L^{\infty}(m)$ we have

$$
\begin{aligned}
\int g u d m & =\lim _{n^{\prime}} \sum_{j=0}^{\infty} a_{n^{\prime}, j} \int\left(T^{j} h\right) u d m \\
& =\lim _{n^{\prime}} \sum_{j=0}^{\infty} a_{n^{\prime}, j} \int\left(T^{j+1} h\right) u d m=\int(T g) u d m .
\end{aligned}
$$

This implies that $g=T g$. Next suppose that $\int(f-T f) u d m=0$ for any $f \in L^{1}(m)$. Then, clearly, $\int\left(f-T^{n} f\right) u d m=0$ for all $n \geqq 0$, and hence

$$
\begin{aligned}
\int g u d m & =\lim _{n^{\prime}} \sum_{j=0}^{\infty} a_{n^{\prime}, j} \int\left(T^{j} h\right) u d m \\
& =\lim _{n^{\prime}} \sum_{j=0}^{\infty} a_{n^{\prime}, j} \int h u d m=\int h u d m .
\end{aligned}
$$

It follows that $h-g$ belongs to the closed linear manifold generated by the set $\left\{f-T f ; f \in L^{1}(m)\right\}$. Thus

$$
\lim _{n}\left\|\frac{1}{n} \sum_{j=0}^{n-1} T^{j} h-g\right\|_{1}=0
$$

and hence $g \geqq 0$. Let $A=\{x \in X ; g(x)=0\}$, and let the conservative and dissipative parts [3] of $T$ be $C$ and $D$, respectively. We shall first prove that $A=D$. It is clear that $D \subset A$. To see that $A \subset D$, let $T^{*}$ denote the corresponding adjoint operator on $L^{\infty}(m)$. Since $T g=g$, it follows that $T^{*} 1_{\mathcal{A}} \leqq 1_{A}$, whence if we define $B=A \cap C$ then $T^{* j} 1_{13}=1_{B}$, almost everywhere on $C$ for each $j \geqq 0$. Thus

$$
\begin{aligned}
\int h 1_{B} d m & \leqq \lim _{n} \int \frac{1}{n} \sum_{j=0}^{n-1} h T^{* j} 1_{B j} d m \\
& =\lim _{n} \int\left(\frac{1}{n} \sum_{j=0}^{n-1} T^{j} h\right) 1_{D^{j}} d m=\int g 1_{B j} d m=0 .
\end{aligned}
$$

Since $h$ is strictly positive, it follows that $m(B)=0$, and hence $A \subset D$.

Let $f$ be any function in $L^{1}(m)$. Since $A=D$, it follows at once that the limit (4) exists and is finite almost everywhere on $A$. On the other hand, the Chacon-Ornstein theorem [4] implies that

$$
\lim _{n} \frac{1}{n} \sum_{j=0}^{n-1} T^{j} f(x)=g(x) \operatorname{iim}_{n}\left(\sum_{j=0}^{n-1} T^{j} f(x) / \sum_{j=0}^{n-1} T^{j} g(x)\right)
$$

exists and is finite almost everywhere on $X-A$. This completes the proof of the theorem. 
It should be pointed out here that if $a_{n, j}$ is a regular matrix such that

$$
\lim _{k \rightarrow \infty} \sum_{j=k}^{\infty}\left|a_{n, j+1}-a_{n, j}\right|=0
$$

uniformly in $n$ then it satisfies (1), (2) and (3) (see [5]).

REMARK 1 . Let $\left\{w_{n} ; n \geqq 1\right\}$ be a sequence of nonnegative numbers whose sum is one, and let $\left\{u_{n} ; n \geqq 0\right\}$ be the sequence defined by $u_{n}=$ $w_{1} u_{n-1}+\cdots+w_{n} u_{0}, u_{0}=1$. Then the above argument together with Baxter's ergodic theorem [2] implies that under the same condition as in the theorem, for any $f \in L^{1}(m)$ the limit

$$
\lim _{n}\left(\sum_{j=0}^{n-1} u_{j} T^{j} f(x) / \sum_{j=0}^{n-1} u_{j}\right)
$$

exists and is finite almost everywhere. The theorem is a special case of this result.

REMARK 2. If $T$ maps, in addition, $L^{p}(m)$ into $L^{p}(m)$ and $\|T\|_{p} \leqq 1$ for some $p$ with $p>1$, then for any $f \in L^{1}(m)$ the limit (5) exists and is finite almost everywhere. This follows from [1] and [7].

\section{BIBLIOGRAPHY}

1. M. A. Akcoglu and R. V. Chacon, A convexity theorem for positive operators, Z. Wahrscheinlichkeitstheorie und Verw. Gebiete 3 (1965), 328-332. MR 32 \#192.

2. G. Baxter, A general ergodic theorem with weighted averages, J. Math. Mech. 14 (1965), 277-288. MR 30 \#4904.

3. R. V. Chacon, Identification of the limit of operator averages, J. Math. Mech. 11 (1962), 961-968. MR 26 \#2883.

4. R. V. Chacon and D. S. Ornstein, A general ergodic theorem, Illinois J. Math. 4 (1960), 153-160. MR 22 \#1822.

5. L. W. Cohen, On the mean ergodic theorem, Ann. of Math. (2) 41 (1940), 505-509.

6. Y. Ito, Uniform integrability and the pointwise ergodic theorem, Proc. Amer. Math. Soc. 16 (1965), 222-227. MR 30 \#2121.

7. R. Sato, On a general ratio ergodic theorem with weighted averages, Proc. Amer. Math. Soc. 35 (1972), 177-178.

Josai University, Sakado, Saitama 350-02, Japan 